\title{
VIRTUAL DAMPING IN OSCILLATORS
}

\author{
Donhee Ham and Ali Hajimiri
}

\author{
California Institute of Technology, Pasadena, CA 91125, USA
}

\begin{abstract}
This paper presents a new point of view of oscillator noise, bringing transparent insight into the phase noise. This work bridges fundamental physics of noise and existing oscillator phase noise theories and reveals the direct correspondence of phase noise and the Einstein relation. The concept of virtual damping is presented utilizing an ensemble of oscillators as a measure of phase noise. The explanation of the linewidth narrowing through virtual damping results in a clear definition of loaded and unloaded quality factors of an oscillator. The validity of this new approach is verified by excellent experimental agreement.
\end{abstract}

\section{INTRODUCTION}

Oscillator phase noise has been studied from several different angles, ranging from a pure mathematical physics treatment [1] to CAD-oriented methods [2] [3] and design-oriented approaches [4] [5], to name a few. The design-oriented approaches have evolved from a familiar linear time-invariant theory [4] to a more accurate time-varying theory [5], adding additional insight into the oscillator design. These theories have helped circuit designers understand the evolution of noise in oscillators, leading to more accurate phase noise predictions, and lower noise designs. However, they assume rather phenomenological standpoints and a more fundamental, yet intuitive understanding of the phase noise phenomenon is still needed. Work in [6] partially addresses the issue but its focus remains on the practical side of oscillator optimization and hence such fundamental physical argument is still lacking.

This paper presents a theoretical work with a supporting measurement that fills in the gap between the fundamental physics of noise and the existing phase noise theories. The work consists of two main contributions. First, using an ensemble of identical oscillators as a tool, we derive the concept of virtual damping, which will enable us to view oscillators and resonators in a unified framework. It will be shown that the virtual damping rate is a fundamental measure of phase noise which can be verified experimentally. Second, we will show that the virtual damping rate can be obtained from the Einstein relation without resorting to specific circuit parameters. The derivation of the virtual damping rate using this method will clearly show the true physical meaning of phase noise by identifying its two essential elements: sensitivity and friction (energy loss), demonstrating good agreement with the existing phase noise theories.

In Section 2, we define the concept of virtual damping and demonstrate phase noise as its natural outcome using both theoretical and experimental treatments. In Section 3, we derive the virtual damping rate based on physical arguments using the Einstein relation and compare the results with the existing theories for both time-invariant and time-varying cases. Finally, we calculate the ratio of the resonator's linewidth to the oscillator's linewidth using virtual damping in Section 4.

\section{VIRTUAL DAMPING}

\subsection{Definition and Qualitative Treatment}

The left hand side of Fig. 1 shows a parallel $L C$ resonator with an effective parallel tank resistance, $R$, representing energy loss.
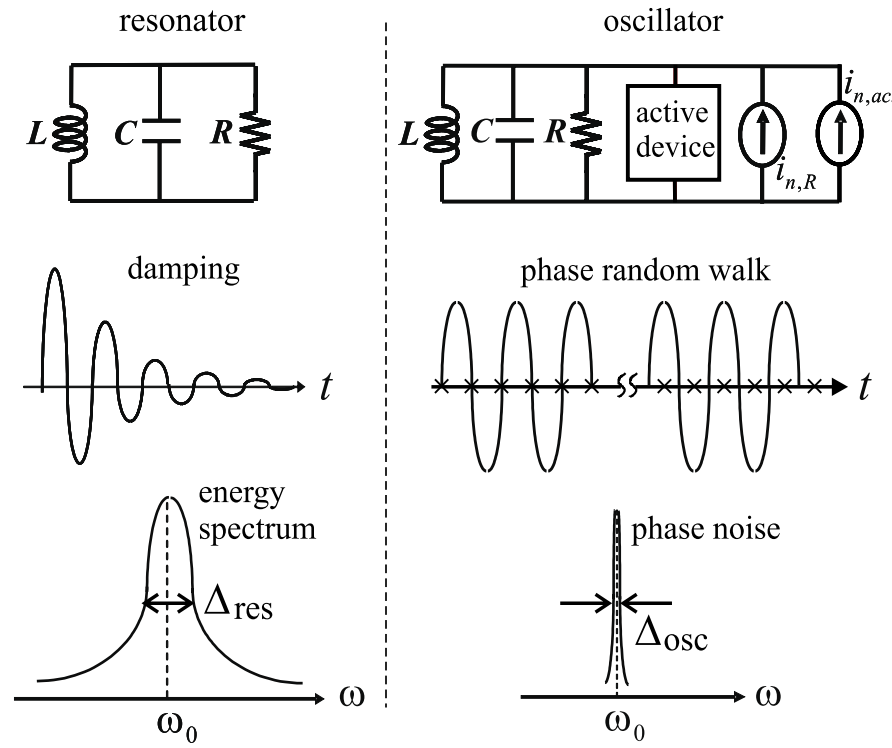

Figure 1: Resonator vs. oscillator. The X's on the $t$-axis represent the ideal zero-crossings.

Due to the loss, the voltage $v$ across the tank shows an exponential damping from a given initial voltage as shown in the figure. This damping corresponds to a Lorentzian line broadening in the energy spectrum.

A negative resistance can be used to cancel these losses to obtain a self-sustained oscillator, as shown in the right hand side of Fig. 1. The active and passive device noise perturbs the phase of the oscillator, resulting in phase random walk or diffusion, which corresponds to a line broadening of oscillator's output power spectral density, or phase noise [1]. The line broadening of the oscillator is much smaller than that of the resonator yet it still has the Lorentzian shape.

A time-domain picture of the phase diffusion is shown in Fig. 2 for an ensemble of $N$ identical oscillators where their initial phase is the same. At the start of oscillation, they have the same phase and the ensemble average $\langle v(t)\rangle$ of the oscillator output $v(t)$ is equal to $v(t)$ of any single oscillator in the ensemble. After a sufficiently long time, however, the output voltages from the ensemble become incoherent due to the phase diffusion (a.k.a., jitter accumulation) and $\langle v(t)\rangle$ tends to zero with time, as shown in Fig. 2 [7]. We will refer to this damping of the ensemble average as virtual damping. In other words, even though the single oscillator output $v(t)$ per se sustains itself, its ensemble average which matters in the measurement of phase noise virtually damps. As we will show in the next subsection, this damping has an exponential behavior. One can logically conceive that the phase diffusion constant should be identical to the virtual damping rate and we will denote this quantity as $D$ from this point on. A lower phase noise implies a smaller phase diffusion constant or a slower virtual damping rate. Hence, the oscillator phase noise can be solely characterized by $D$.

This virtual damping manifests itself in the autocorrelation function as well. $v\left(t_{1}\right) v\left(t_{2}\right)$ from the ensemble grows incoherent due to the phase diffusion after a large enough time delay $\left|t_{1}-t_{2}\right|$ and hence the autocorrelation function $\left\langle v\left(t_{1}\right) v\left(t_{2}\right)\right\rangle$ tends to zero with 


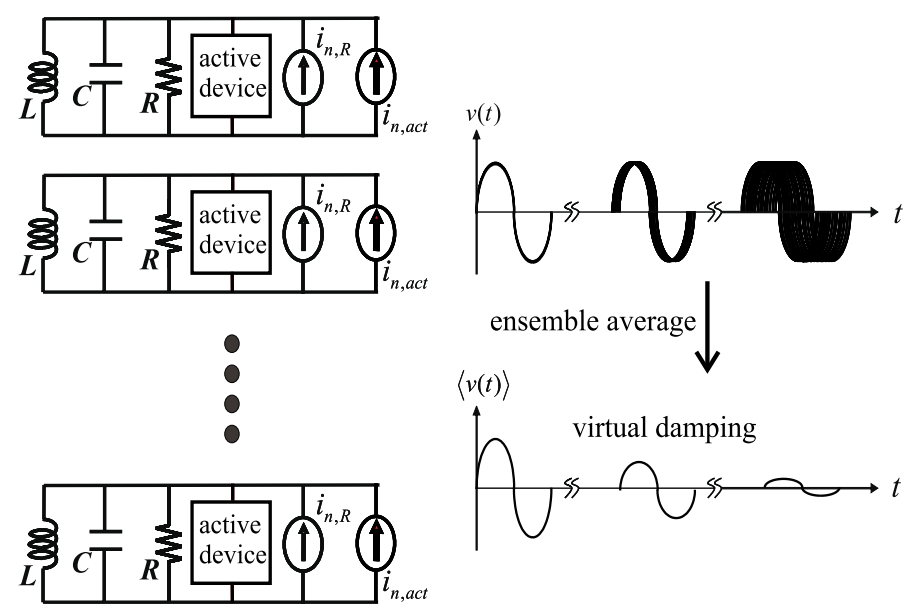

Figure 2: Ensemble average of $v(t)$ and virtual damping

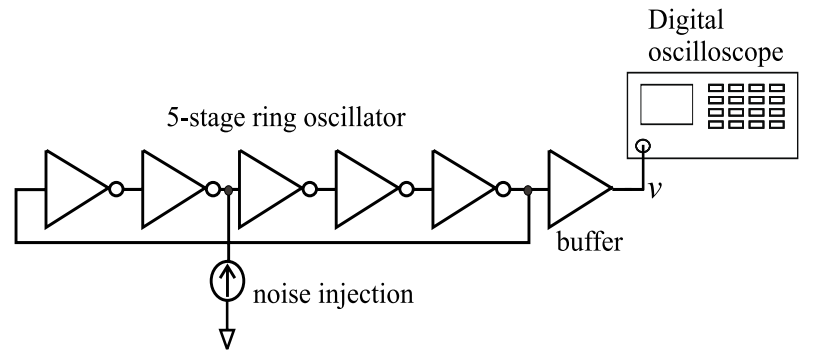

Figure 3: Measurement setup for the virtual damping using a digital oscilloscope.

an increasing time difference $\left|t_{1}-t_{2}\right|$, again showing virtual damping.

We can observe the virtual damping phenomenon experimentally, too. As will be seen later, typical oscillators have very slow virtual damping rates making them less suitable for experimental verification. Instead, we use a ring oscillator whose phase noise is degraded by injection of a white noise current whose power spectrum can be controlled externally. This setup is shown in Fig. 3. The oscillator has a center frequency of $5 \mathrm{MHz}$. A digital oscilloscope is used to sample the output waveform multiple times and calculate the average over $N$ samples $\langle v(t)\rangle_{N}$. Fig. 4 shows this average for $N=512$ samples as a function of time. As can be seen clearly, the expected value of the output is an exponentially damping sinusoidal even though the output waveform is a steady-state sinusoidal in complete agreement with the virtual damping concept.

The spectral line broadening of an oscillator output signal or phase noise can be thought of as the result of the virtual damping and this provides an explanation why both resonator's energy spectrum and oscillator's output power spectral density have Lorentzian broadening with different linewidths. Since the virtual damping rate is much slower than the damping in the resonator, the linewidth of

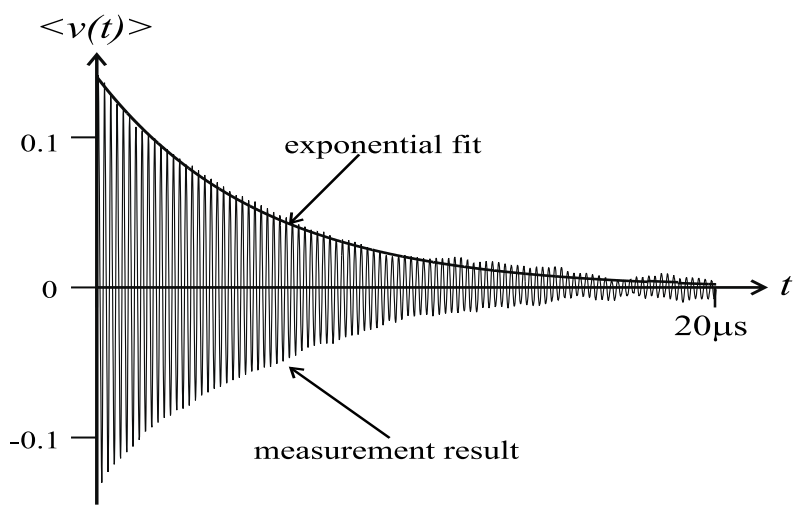

Figure 4: Measured $\langle v(t)\rangle_{512}$ vs. $t$ for a $5 \mathrm{MHz}$ ring oscillator.

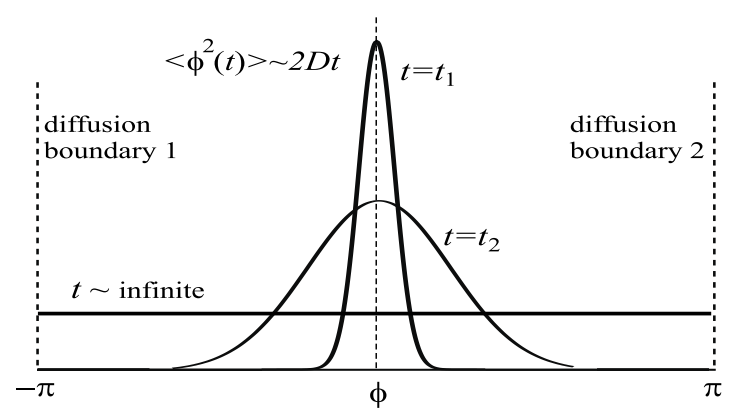

Figure 5: Time evolution of phase distribution

the oscillator output spectrum is much smaller than the linewidth of the resonator's energy spectrum, as shown hypothetically in Fig. 1. In other words, placing a resonator in a positive feedback loop for oscillation results in the linewidth narrowing. The quantitative calculation of the linewidth narrowing will be given in Sec. 4 .

Fig. 5 shows time evolution of phase distribution for the ensemble of oscillators whose initial phases are given at 0 . The phase distribution will ultimately tend to a uniform distribution across the whole range of phase $[-\pi, \pi]$ since there is no physical mechanism to restore the initial phase and the uniform distribution constitutes the most probable (the maximum entropy) state. Hence the virtual damping rate or the phase diffusion constant $D$ is a direct measure of how fast the entropy grows. This implies that the minimization of phase noise is equivalent to minimization of the entropy-growth-rate.

\subsection{Quantitative Treatment}

A mathematical verification of the virtual damping is given in this subsection. The output voltage $v(t)$ of an oscillator without amplitude variation can be expressed as

$$
v(t)=r_{0} \cos \left[\omega_{0} t+\phi(t)\right]
$$

where $r_{0}, \omega_{0}$ and $\phi(t)$ are the voltage amplitude, oscillation frequency and phase noise, respectively. In the presence of white noise, the phase noise $\phi(t)$ becomes a Wiener process (diffusion) where $\left\langle\phi^{2}(t)\right\rangle=2 D t$ with diffusion constant $D$ [1] [8] [9]. For a Gaussian distribution of $\phi(t)$ at any given time, $t$, we have $\langle\cos \phi\rangle=e^{-\left\langle\phi^{2}\right\rangle / 2}$ and $\langle\sin \phi\rangle=0$ and therefore

$$
\langle v(t)\rangle=r_{0} e^{-\left\langle\phi^{2}(t)\right\rangle / 2} \cos \left(\omega_{0} t\right)=r_{0} e^{-D t} \cos \left(\omega_{0} t\right)
$$

which clearly shows the exponential behavior of virtual damping, the rate of which is given by the diffusion constant $D$. Similarly, one can show

$$
\langle v(t) v(t+\tau)\rangle=\frac{1}{2} r_{0}^{2} e^{-D|\tau|} \cos \omega_{0} \tau
$$

which represents the virtual damping of the autocorrelation.

The power spectral density of the oscillator output is the Fourier transform of the autocorrelation function (3) and thus results in the familiar Lorentzian shape [1]

$$
S_{v, v}(f)=r_{0}^{2} \frac{D}{D^{2}+(\Delta \omega)^{2}}
$$

where $\Delta \omega \equiv \omega-\omega_{0}$. As $D$ becomes larger, the Lorentzian shape becomes shorter and fatter, distributing the total energy of $r_{0}^{2} / 2$ further from the center frequency, degrading the phase noise. This is the frequency domain meaning of the virtual damping rate $D$. For $\Delta \omega \gg D$, the phase noise assumes a familiar $f^{-2}$ behavior:

$$
\mathcal{L}\{\Delta \omega\}=\frac{S_{v, v}(f)}{r_{0}^{2} / 2} \approx \frac{2 D}{(\Delta \omega)^{2}}
$$

As a numerical example, a $1 \mathrm{GHz}$ oscillator whose phase noise is $-121 \mathrm{dBc} / \mathrm{Hz}$ at $600 \mathrm{kHz}$ offset has $D \approx 5.645$ or $D / \omega_{0} \sim 10^{-9}$. As can be seen, typical good oscillators have very slow virtual damping rates when compared to oscillation frequencies. 


\subsection{Experimental Verifications}

Using the experimental setup of Fig. 3, the virtual damping rate, $D$, (reciprocal of the exponential time constant) was measured for different injected noise power levels. $D$ is the inverse of time constant of the best-fit exponential to the resultant time domain waveforms (e.g., Fig. 4). The oscillator phase noise was also measured using a spectrum analyzer at $1 \mathrm{MHz}$ offset from the carrier. Equation (5) was used to predict the phase noise using the virtual damping rate $D$. The results are summarized in Table 1 showing very good agreements between the two methods.

\begin{tabular}{c|c|c|c}
\hline $\begin{array}{c}\overline{i_{n}^{2} / \Delta f} \\
\left(A^{2} / \mathrm{Hz}\right)\end{array}$ & $\begin{array}{c}D \\
\left(\mathrm{sec}^{-1}\right)\end{array}$ & $\begin{array}{c}\text { PN from } \\
\text { measured } D \\
(\mathrm{dBc} / \mathrm{Hz})\end{array}$ & $\begin{array}{c}\text { PN from } \\
\text { spec. analyzer } \\
(\mathrm{dBc} / \mathrm{Hz})\end{array}$ \\
\hline \hline $2.60 \times 10^{-15}$ & $1.02 \times 10^{4}$ & -92.9 & -93.0 \\
\hline $4.84 \times 10^{-15}$ & $1.56 \times 10^{4}$ & -91.0 & -90.0 \\
\hline $9.66 \times 10^{-15}$ & $3.53 \times 10^{4}$ & -87.4 & -86.5 \\
\hline $2.12 \times 10^{-14}$ & $9.30 \times 10^{4}$ & -83.3 & -81.7 \\
\hline $6.04 \times 10^{-14}$ & $1.90 \times 10^{5}$ & -80.0 & -79.5 \\
\hline
\end{tabular}

Table 1. Measured $D$, phase noise calculated from the measured $D$, and phase noise measured using a spectrum analyzer. The offset frequency is $1 \mathrm{MHz}$ and the center frequency of the oscillator is $5 \mathrm{MHz}$.

\section{PHASE NOISE AS EINSTEIN RELATION}

In this section, we will determine the virtual damping rate, $D$, using a fundamental argument based on the theory of diffusion. The key to this approach is the notion that the rate of any diffusion process is determined by two elements affecting the process: the sensitivity of the physical quantity undergoing the diffusion and the friction (energy loss) of the environment in which the diffusion process occurs.

For example, the diffusion constant, $D$, of a Brownian particle of mass, $M$, immersed in a liquid at temperature, $T$, with frictional coefficient of $\gamma$ in Fig. 6 is given by the Einstein relation [10] ${ }^{1}$ :

$$
D=\frac{k_{B} T}{M} \cdot \frac{1}{\gamma}
$$

where $\gamma$ determines the frictional force of $M \gamma V$ for a Brownian particle with velocity of $V$. The $k_{B} T / M$ factor represents the sensitivity of the Brownian particle to perturbations and becomes smaller with a larger mass. This factor is obtained using the equipartition theorem of statistical physics [12] demanding that each independent degree of freedom of a system in equilibrium at temperature $T$ has a mean energy of $k_{B} T / 2$, i.e., $\left\langle M V^{2} / 2\right\rangle=k_{B} T / 2$. This sensitivity factor is independent of the friction coefficient of the liquid. Also, if two identical Brownian particles are immersed in liquids with different frictions, the Brownian particle in a medium with more friction will exhibit a slower diffusion, and hence the second factor $1 / \gamma$ in (6). Summarizing, the diffusion constant can be determined only when both sensitivity and friction (energy loss) elements are known. Now by applying the same concept to the electrical oscillators, we will quantify the phase diffusion, starting with the simpler time-invariant case.

\subsection{Time-Invariant Case}

Fig. 7 shows the oscillation trajectory (limit cycle) in the $\left(v, \dot{v} / \omega_{0}\right)$ state space for an ideal sinusoidal oscillator output. ${ }^{2,3}$ A phase random walk by the amount of $\Delta \phi$ corresponds to a random walk of the oscillation trajectory point from $\mathbf{A}_{1}$ to $\mathbf{A}_{2}$ or equivalently a voltage random walk from $\mathbf{B}_{\mathbf{1}}$ to $\mathbf{B}_{\mathbf{2}}$. In the following, we will first characterize this voltage diffusion and conversion to the phase diffusion can be easily performed by a multiplicative factor $1 / r_{0}^{2}$.

\footnotetext{
${ }^{1}$ In semiconductor physics, this is usually expressed as $D / \mu=k_{B} T / e$ where $\mu$ is the mobility [11].

${ }^{2}$ The dot signifies a time-derivative.

$31 / \omega_{0}$ is used to keep the unit of voltage for the 2 nd variable.
}
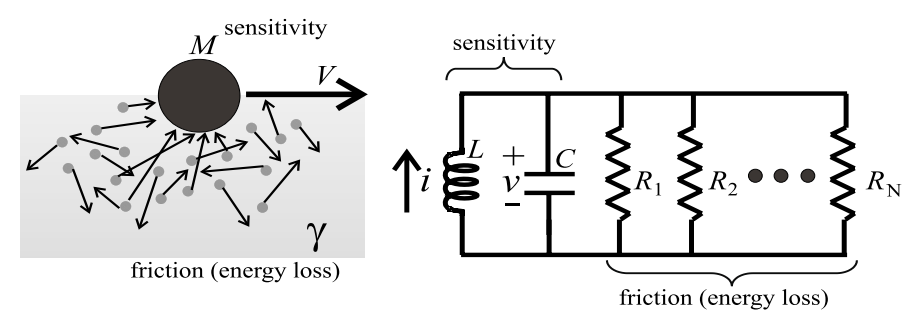

Figure 6: Brownian particle and $L C$ tank with losses.

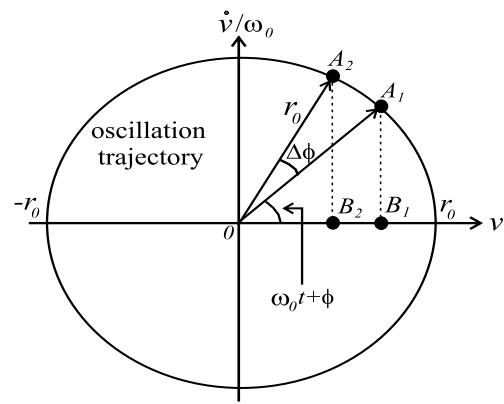

Figure 7: Oscillation trajectory in state space and phase diffusion

According to the fluctuation-dissipation theorem of statistical physics [12] that states the equivalence of a loss (dissipation) element and a thermal noise (fluctuation) element, any thermal noise source in a circuit can be replaced with a loss. The right hand side of Fig. 6 shows a parallel $L C$ tank with multiple parallel losses in a given oscillator that represent the noise sources. $R_{1}$ is always reserved for the effective parallel tank resistance. A shortchannel MOS transistor in parallel with the $L C$ tank will contribute a loss element $R=1 /\left(\gamma_{\operatorname{tran}} g_{d 0}\right)$ as its noise contribution is $\overline{i_{n}^{2} / \Delta f}=4 k_{B} T \gamma_{\operatorname{tran}} g_{d 0}$ where $\gamma_{\text {tran }}$ is the CMOS thermal noise factor and $g_{d 0}$ is the transconductance at $V_{d s}=0$. Note that Fig. 6 is not an equivalent circuit model for the oscillator but is used to identify the sensitivity and friction parts of the voltage diffusion as in the following.

In the circuit of Fig. $6,\left\langle v^{2}\right\rangle$ across the capacitor and $\left\langle i^{2}\right\rangle$ through the inductor can be calculated noting that each of them constitutes a degree of freedom which will have $k_{B} T / 2$ of thermal energy according to equipartition theorem: $\left\langle v^{2}\right\rangle=k_{B} T / C$ and $\left\langle i^{2}\right\rangle=k_{B} T / L$. This is analogous to the $k_{B} T / M$ sensitivity factor of the Brownian motion. Just as the sensitivity of the Brownian particle was described in terms of the velocity, $V$ (time-derivative of the displacement, $X$, that diffuses), the sensitivity of the voltage diffusion is to be described in terms of its time-derivative, $\dot{v}$, i.e.:

$$
\left\langle\dot{v}^{2}\right\rangle=\frac{1}{C^{2}}\left\langle i^{2}\right\rangle=\frac{k_{B} T}{L C^{2}}=\frac{k_{B} T}{C} \omega_{0}^{2} \quad \text { [sensitivity factor] }
$$

The friction (energy loss) associated with the oscillator can be calculated from the $L R$ part of the resonator. Since the current in the circuit satisfies $i=-\left(L / R_{e q}\right) \dot{i}$ where $R_{e q} \equiv R_{1}\left\|R_{2}\right\| \cdots \| R_{N}$ in the absence of the capacitor, similar to the equation of motion $\dot{V}=$ $-\gamma V$ for the Brownian motion, we can see the friction coefficient $\gamma$ of the system is given by

$$
\left.\frac{1}{\gamma}=\frac{L}{R_{e q}} \quad \text { [energy loss (friction) factor }\right]
$$

Now taking both sensitivity and loss into account and using the Einstein's relation, the phase diffusion constant $D$ is given by

$$
D=\frac{1}{r_{0}^{2}} \cdot\left\langle\dot{v}^{2}\right\rangle \cdot \frac{1}{\gamma}=\frac{1}{r_{0}^{2}} \cdot \frac{k_{B} T}{C} \cdot \frac{\omega_{0}}{Q_{\text {loaded }}}
$$

where the factor $1 / r_{0}^{2}$ was introduced to convert the voltage diffusion to the phase diffusion as mentioned earlier and $Q_{\text {loaded }} \equiv C R_{e q} \omega_{0}$. Since $Q_{\text {loaded }}=\omega_{0} E_{\text {tank }} / P_{\text {sig }}=\omega_{0} C r_{0}^{2} /\left(2 P_{\text {sig }}\right)$ where $P_{\text {sig }}$ is the power dissipation in the resonator, (9) can be rewritten as

$$
D=\frac{k_{B} T}{2 P_{\text {sig }}} \cdot\left(\frac{\omega_{0}}{Q_{\text {loaded }}}\right)^{2}
$$


Combining this with (5) leads to Leeson's formula in the $1 / f^{2}$-region except for the fitting parameter $F / 2[4]$.

Summarizing, by evaluating the sensitivity and energy loss (friction) factors of the phase diffusion process, we derived the virtual damping constant and hence, phase noise. The power of this approach lies in the identification of the two essential elements of the oscillator phase noise. The importance of the largely-ignored sensitivity factor $k_{B} T / C$ has been noted in [6] from a practical perspective.

\subsection{Time-Varying Case}

In the foregoing argument, we ignored the time-varying effects in the phase noise evaluation. In actuality, the phase $\phi(t)$ of an oscillator experiences a time-varying diffusion in that the average size of the phase random walk periodically changes in the course of oscillation [5]. The time-varying effects are quantified using the $\mathrm{im}$ pulse sensitivity function $\Gamma(t)$ describing the periodic sensitivity of the oscillator phase to the perturbation and the noise modulating function (NMF) $\alpha(t)$ accounting for the cyclostationary noise generation [5]. This separation of the time-varying effects into the ISF and the NMF agrees perfectly with our earlier view of separating the phase diffusion into the sensitivity and loss (friction) part. The ISF affects the sensitivity part and modulates (7) periodically, i.e., $\left\langle\dot{v}^{2}\right\rangle=\left(k_{B} T / C\right) \omega_{0}^{2} \Gamma^{2}(t)$. On the other hand, the NMF describing the cyclostationary noise is equivalent to periodic circuit loss modulation in the course of oscillation, i.e., $1 / \gamma=\left(L / R_{e q}\right) \alpha^{2}(t)$.

Now we will modify our previous derivation of the virtual damping constant $D$ to incorporate the time-varying effects. The timevarying effects modulate the average size of the random walk in the course of oscillation. Since the phase variance $\left\langle\phi^{2}(t)\right\rangle$ in the diffusion process is an accumulation of this time-varying random walk over time, after a long observation time, the details of time-variance in $\left\langle\phi^{2}(t)\right\rangle$ will become negligible, hidden behind the $2 D t$ diffusion where the diffusion constant is now affected by $\Gamma(t)$ and $\alpha(t)$ in an averaged sense, modifying the contribution from the $n$-th noise source $R_{n}$ in (9) to

$$
D_{n}=\frac{1}{r_{0}^{2}} \cdot \frac{k_{B} T}{C} \cdot \frac{\Gamma_{e f f, r m s, n}^{2}}{R_{n} C}
$$

where $\Gamma_{e f f, n}(t)=\Gamma_{n}(t) \alpha_{n}(t)$. Now including all the noise sources, we have

$$
D=\sum_{n} D_{n}=\frac{1}{r_{0}^{2}} \cdot \frac{k_{B} T}{C} \cdot \frac{\omega_{0}}{Q_{\text {loaded }}}
$$

where the new definition for $Q_{\text {loaded }}$ is

$$
Q_{\text {loaded }} \equiv \omega_{0} C\left[\frac{R_{1}}{\Gamma_{\text {eff }, r m s, 1}^{2}}\|\cdots\| \frac{R_{N}}{\Gamma_{\text {eff,rms }, N}^{2}}\right]
$$

Using $q_{\max }=r_{0} C$ and $\overline{i_{n}^{2} / \Delta f}=4 k_{B} T / R_{n}, D_{n}$ in (11) can be rewritten as

$$
D_{n}=\frac{\Gamma_{e f f, r m s, n}^{2}}{4 q_{\max }^{2}} \cdot \overline{\frac{i_{n}^{2}}{\Delta f}} .
$$

and the combination of (14), (12) with (5) lead us to

$$
\mathcal{L}\{\Delta \omega\}=\sum_{n} \frac{\overline{i_{n}^{2} / \Delta f} \cdot \Gamma_{e f f, r m s, n}^{2}}{2 q_{\max }^{2}(\Delta \omega)^{2}}
$$

in complete agreement with the time-varying phase noise theory in $[5]$.

\section{LINEWIDTH NARROWING -RESONATOR VS. OSCILLATOR-}

We can compare the linewidth of the oscillator to that of the resonator in Fig. 1 as the damping rate determines the linewidth of the frequency spectrum. This comparison will reveal how much linewidth improvement we obtain by placing a given resonator into a feedback loop and making an oscillator. Since the damping rate for the resonator is given by $1 /(2 R C)$, we define a linewidth narrowing ratio $S$ as

$$
S \equiv \frac{\Delta_{o s c}}{\Delta_{\text {res }}}=\frac{D_{\text {osc }}}{D_{\text {res }}}=\frac{2}{r_{0}^{2}} \cdot \frac{k_{B} T}{C} \cdot \frac{Q_{\text {unloaded }}}{Q_{\text {loaded }}}
$$

where we have used $Q_{\text {unloded }}=R C \omega_{0}$ and (12). In a typical electrical oscillator at a normal temperature, this ratio is extremely small: for instance, for $C=1 p F, r_{0}=1 \mathrm{~V}, Q_{\text {unloaded }}=10$ and $Q_{\text {loaded }}=5, S \approx 1.6 \times 10^{-8}$ and hence shows that the linewidth of a resonator is narrowed by almost 8 orders of magnitude when placed in a positive feedback loop to make an oscillator.

One important point is that the linewidth narrowing is directly proportional to the ratio of $k_{B} T / C$ to oscillator mean square amplitude. This shows the crucial importance of selecting a larger tank amplitude and a larger $C$ (or a smaller $L$ ) for given quality factors to improve the phase noise [6].

\section{CONCLUSION}

In this paper, we presented a theoretical framework with a supporting measurement that views the oscillator phase noise from a fundamental physics perspective. The virtual damping concept puts the oscillator phase noise theory and well-known resonator theory under the same framework. We have shown that the virtual damping rate is identical to the phase diffusion constant and hence a direct measure of the oscillator phase noise. Identification of oscillator phase noise as the Einstein relation reveals the essence of oscillator phase noise from fundamental physics perspective, bringing a transparent insight into the noise phenomenon.

\section{ACKNOWLEDGMENT}

Authors would like to thank B. Analui, H. Hashemi, A. Komijani and C. White of Caltech for their valuable suggestions and helpful discussions. We also acknowledge the support of this project by ONR Grant (N00014-01-1-0764), IBM Corporation, National Science Foundation, and Lee Center for Advanced Networking.

\section{REFERENCES}

[1] M. Lax, "Classical noise. V. noise in self-sustained oscillators," Phys. Rev., vol. CAS-160, pp. 290-307, 1967.

[2] F. K. Kartner, "Analysis of white and $f^{-\alpha}$ noise in oscillators," Int. J. Circuit Theory Appl., vol. 18, pp. 485-519, 1990.

[3] A. Demir, A. Mehrotra, and J. Roychowdhury, "Phase noise in oscillators: a unifying theory and numerical methods for characterization," IEEE Trans. on Circuits and Systems-I: Fundamental theory and appl., vol. 47, no. 5, May 2000.

[4] D. B. Leeson, "A simple model of feedback oscillator noise spectrum," Proc. IEEE, vol. 54, pp. 329-330, Feb. 1966.

[5] A. Hajimiri and T. H. Lee, "A general theory of phase noise in electrical oscillators," IEEE Solid-State Circuits, vol. 33, pp. 179-194, Feb. 1998.

[6] D. Ham and A. Hajimiri, "Concepts and methods in optimization of integrated LC VCOs," IEEE Solid-State Circuits, vol. 36, pp. 896909, June, 2001.

[7] P. Grivet and A. Blaquiere, "Nonlinear effects of noise in electronic clocks," Proceedings of the IEEE, pp. 1606-1614, vol. 51, no. 11, November 1963.

[8] J. A. McNeill, "Jitter in ring oscillators," Ph.D. dissertation, Boston Univ., 1994.

[9] A. Hajimiri, S. Limotyrakis and T. H. Lee, "Jitter and phase noise of ring oscillators," IEEE Solid-State Circuits, vol. 34, pp. 790-804, June 1999.

[10] A. Einstein, Investigation on the Theory of the Brownian Motion, Dover Publication, 1956.

[11] S. M. Sze, Physics of Semiconductor Devices, 1981, John Wiley \& Sons.

[12] F. Reif, "Fundamentals of Statistical and Thermal Physics," 1985, McGraw-Hill. 